\title{
A Phase I Study of Combination Therapy with Sorafenib and 5- Fluorouracil in Patients with Advanced Hepatocellular Carcinoma
}

\author{
Takuya Sho $^{1} \cdot$ Mitsuru Nakanishi $^{1,5} \cdot$ Kenichi Morikawa $^{1}$ (D) Masatsugu Ohara $^{1} \cdot$ \\ Naoki Kawagishi $^{1}$ - Takaaki Izumi ${ }^{1}$ - Machiko Umemura ${ }^{1} \cdot$ Jun Ito $^{1}$ • \\ Masato Nakai $^{1}$ - Goki Suda ${ }^{1} \cdot K_{0 j i}$ Ogawa $^{1} \cdot$ Makoto Chuma $^{1,6}$ - Takashi Meguro ${ }^{2}$. \\ Michio Nakamura $^{3} \cdot$ Atsushi Nagasaka $^{3} \cdot$ Hiromasa Horimoto $^{4} \cdot$ Yoshiya Yamamoto $^{4}$. \\ Naoya Sakamoto ${ }^{1}$
}

Published online: 1 June 2017

(C) The Author(s) 2017. This article is an open access publication

\begin{abstract}
Background and aims Sorafenib is the first molecular targeted drug approved for the treatment of advanced hepatocellular carcinoma (HCC) and is a potent small molecule inhibitor of multiple kinases. Combination therapy with sorafenib and other cytotoxic agents for HCC may result in additive anticancer activity. The purpose of this phase I study was to investigate the safety and tolerability of combination therapy with sorafenib and 5-fluorouracil (5-FU) and to determine the optimum dose of 5-FU for a phase II trial.

Methods This phase I study used a conventional $3+3$ dose-escalation design. The primary endpoint was to determine the maximum tolerated dose (MTD) of 5-FU in combination with sorafenib and to determine the recommended dosage (RD) for phase II. The secondary endpoints evaluated were toxicity and the tumor response rate. All patients received $800 \mathrm{mg}$ of sorafenib daily and three
\end{abstract}

Takuya Sho and Mitsuru Nakanishi contributed equally to this work.

Kenichi Morikawa

kenichi.morikawa@med.hokudai.ac.jp

1 Division of Gastroenterology and Hepatology, Hokkaido University Hospital, Kita 15 Nishi 7, Kita-ku, Sapporo, Hokkaido 060-8638, Japan

2 Internal Medicine, Hokkaido Shokakika Hospital, Sapporo, Hokkaido, Japan

3 Department of Gastroenterology, Sapporo City General Hospital, Sapporo, Hokkaido, Japan

4 Department of Gastroenterology, Hakodate Municipal Hospital, Hakodate, Hokkaido, Japan

5 Present Address: Kotoni Family Clinic, Sapporo, Hokkaido, Japan

6 Present Address: Gastroenterological Center, Yokohama City University Medical Center, Yokohama, Kanagawa, Japan different dosages of 5 -FU (250, 350, and $450 \mathrm{mg} / \mathrm{m}^{2} /$ day $)$ for 20 days by intravenous infusion in 1 month as one cycle.

Results Twelve patients with advanced HCC were evaluated. The MTD of 5-FU in combination with sorafenib was $450 \mathrm{mg} / \mathrm{m}^{2} /$ day, and $350 \mathrm{mg} / \mathrm{m}^{2} /$ day was selected as the RD for a phase II study. Thrombocytopenia, stomatitis, and hand-foot skin reaction were observed as grade 3 adverse events. Nine patients achieved stable disease (75\%), and three patients $(25 \%)$ were judged to have progressive disease. The disease control rate was $75 \%$.

Conclusions Combination therapy with sorafenib and 5-FU appears to be well tolerated and may have the potential to be an option for advanced HCC.

\section{Key Points}

The recommended dosage for the Phase II study was $350 \mathrm{mg} / \mathrm{m}^{2} /$ day in combination with $800 \mathrm{mg}$ of sorafenib daily.

The disease control rate was $75 \%$ in this Phase I study.

Combination therapy with sorafenib and 5-FU appears to be well tolerated and may have the potential of an option for advanced HCC.

\section{Introduction}

Hepatocellular carcinoma (HCC) is the sixth most common cancer and the second leading cause of cancer-related death in the world [1-3]. The prognosis of HCC can be improved by early detection and effective treatment, including 
resection, liver transplantation, transcatheter arterial chemoembolization (TACE), and radiofrequency ablation (RFA). However, HCC patients with portal vein tumor thrombosis (PVTT) or with extrahepatic metastasis have an extremely poor prognosis [4-6]. According to the consensus-based treatment algorithm for HCC proposed by the Japan Society of Hepatology (JSH), treatment should be performed as if extrahepatic spread is negative or extrahepatic spread is not regarded as a prognostic factor [7]. Systemic chemotherapy is sometimes performed when liver function is preserved despite the absence of evidence of survival benefit in all stages of HCC. Systemic chemotherapies with various cytotoxic agents for advanced HCC patients have been tested; the response rates in mono- or combination treatment varied and were unsatisfactory. Most phase III studies failed to demonstrate an improvement in overall survival (OS) [8, 9]. Only a few studies showed improvement in OS in patients with advanced HCC; meanwhile the outcome of liver cancer treatment was different between Eastern and Western patients [10-13]. Currently, there is no standard systemic chemotherapy with cytotoxic agents for advanced HCC patients. However, combination treatment with cytotoxic agents plus molecular targeted agents has the potential for tremendous impact on advanced HCC. The pyrimidine antimetabolite 5-fluorouracil (5-FU) was the first reported chemotherapeutic agent tested in the treatment of HCC, and it has been used with various treatment schedules, dosages, and durations $[8,14,15]$. It has been reported that, when used in combination therapy with 5-FU, several drugs that are modulators that increase 5-FU activity improved the response rate or OS in advanced HCC patients in small trials [16-18].

Sorafenib is the first molecular targeted drug approved for the treatment of advanced HCC and is a potent small molecule inhibitor of multiple kinases, including the serine-threonine kinase Raf-1, the tyrosine kinases, vascular endothelial growth factor receptor (VEGFR), and the platelet-derived growth factor receptor- $\beta$ with anti-proliferative and anti-angiogenic activities. Preclinical studies have shown that sorafenib controls tumor proliferation through inhibition of the MAP kinase cascade and tumor angiogenesis through inhibition of VEGFR [19]. The phase III Sorafenib HCC Assessment Randomized Protocol (SHARP) trial demonstrated prolonged overall and progression-free survival in patients with unresectable HCC who were treated with sorafenib, compared with those who received placebo [20]. A randomized, double-blind, placebo-controlled study in the Asia Pacific region demonstrated the efficacy and safety of sorafenib in advanced HCC, similar to the SHARP study [21]. According to the Barcelona Clinic Liver Cancer (BCLC) staging classification, advanced stage (stage $\mathrm{C}$ ) patients who have vascular invasion or extrahepatic spread but conserved liver function are candidates for systemic therapy with sorafenib [22]. According to the modified form of the original version of the consensus-based treatment algorithm for HCC proposed by the JSH in 2014, sorafenib is the standard of care for patients with an extrahepatic lesion or with TACErefractory or TACE-failure disease in Japan [7]. However, the antitumor effects are insufficient for controlling the large and numerous nodules of HCC and for preventing a high rate of tumor recurrence after treatment [23-25]. A series of clinical studies on the molecular targeted drugs for HCC such as brivanib [26, 27], sunitinib [28], linifanib [29], ramucirumab [30], everolimus [31], and erlotinib [32] has been conducted. No molecular targeted drugs other than sorafenib significantly improve OS in patients with advanced HCC; so far sorafenib is still standard treatment for advanced HCC.

It was previously reported that the serum VEGF level correlated with tumor progression and poor prognosis $[33,34]$. In addition to its direct anti-angiogenic effects, these VEGFR-targeted agents may enhance chemotherapy by normalizing tumor vasculature and decreasing the elevated interstitial pressure in tumors [35]. These reports suggest that combination therapy with sorafenib and cytotoxic agents such as 5-FU has the potential to have additive anticancer activity. Petrini et al. reported a phase II study of combination therapy with sorafenib and 5-FU [36]. Their treatment schedule was $800 \mathrm{mg}$ of sorafenib daily and continuous infusion of 5 -FU $\left(200 \mathrm{mg} / \mathrm{m}^{2} /\right.$ day $)$ from day 1 to 14 (21-day cycles), although high-dose 5-FU was used with manageable side effects in previous reports [16-18]. Their results showed that the median time to progression (TTP) was 8 months [95\% confidence interval (CI) 5.7-10.4], and median OS was 13.7 months (95\% CI 9.5-17.9). Therefore, combination therapy with sorafenib and high-dose 5-FU must be tested as a potential treatment option for advanced HCC.

The purpose of this phase I study was to evaluate the safety and tolerability of combination therapy with sorafenib and dose escalation of continuous infusion of 5-FU at three dosage levels $\left(250,350\right.$, and $450 \mathrm{mg} / \mathrm{m}^{2} /$ day $)$, as well as tumor response in patients with advanced HCC.

\section{Materials and Methods}

\subsection{Study Design}

The study was a non-randomized, uncontrolled, non-blinded, phase I study to investigate the safety and tolerability of combination treatment with sorafenib and 5-FU in patients with advanced HCC. The primary endpoint was to determine the maximum tolerated dose (MTD) of 5-FU in combination therapy with sorafenib and to define the 
recommended dosage (RD) for phase II. Secondary endpoints included toxicity and the response rate. Enrollment began in September 2010, and the study closed in September 2011. The study protocol was approved by the Institutional Review Board of Hokkaido University and the ethics committee of Hokkaido University Hospital (IRB010-0099). Written, informed consent to participate in this study was obtained from each patient. All procedures performed in studies involving human participants were in accordance with the ethical standards of the institutional research committee and with the 1964 Helsinki declaration and its comparable ethical standards. This study was registered at the UMIN Clinical Trials Registry as UMIN000004313.

\subsection{Eligibility Criteria}

The eligibility criteria for this study were as follows: (1) patients with advanced HCC confirmed histologically or clinically based on the American Association for the Study of Liver Diseases guidelines [37]; (2) unresectable and incurable with RFA or TACE; (3) age $\geq 20$ years; (4) at least one measurable lesion on computed tomography (CT) or magnetic resonance imaging (MRI) scans (not including necrotic lesions caused by prior treatment); (5) other prior treatments without systemic chemotherapy permitted if such treatment performed at least 28 days before registration; (6) cirrhotic status of Child-Pugh A; (7) Eastern Cooperative Oncology Group performance status of 0 or 1; (8) hematological findings (neutrophil count of $\geq 1500$ cells $/ \mathrm{mm}^{3}$, platelet count of $\geq 50,000$ cells $/ \mathrm{mm}^{3}$, and hemoglobin of $\geq 10.0 \mathrm{~g} / \mathrm{dL}$ ), renal function (serum creatinine concentration of $\leq 1.5 \times \mathrm{ULN}$ ), and hepatic function (total bilirubin level of $\leq 2.0 \mathrm{mg} / \mathrm{dL}$, aspartate and alanine transaminase levels of $\leq 5.0 \times \mathrm{ULN}$ ); and (9) written, informed consent from the patient. Patients who previously received systemic anticancer therapy or molecular target therapy including sorafenib were excluded from this study. Other exclusion criteria were (1) uncontrollable ascites; (2) varices in the esophagus or stomach with a high risk of bleeding; (3) medical history of hepatic coma; (4) clinically evident congestive heart failure, serious cardiac arrhythmias, active or symptomatic coronary artery disease or ischemia; (5) primary and metastatic brain tumors; (6) history of organ allograft; (7) prior malignancy; (8) treatment with biologic-response modifiers or CYP3A4 inhibitors; or (9) medical/psychological/social problems that might affect study participation or evaluations.

\subsection{Treatment Protocol}

This study used a conventional $3+3$ dose-escalation design. All patients received sorafenib $800 \mathrm{mg}$ daily and
Cohort 1

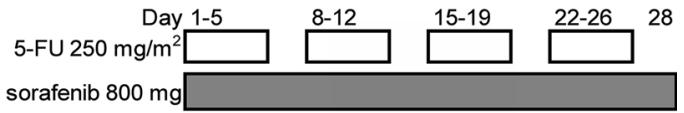

Cohort 2

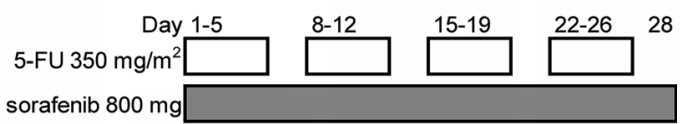

Cohort 3

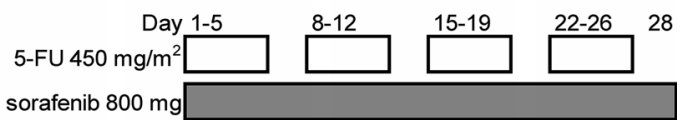

Fig. 1 A $3+3$ dose-escalation study design. Continuous sorafenib $800 \mathrm{mg} /$ day and 3 planned dose levels of $5-\mathrm{FU}$ (250, 350, and $450 \mathrm{mg} / \mathrm{m}^{2} /$ day) for 20 days (day $1-5,8-12,15-19$, and 22-26) by intravenous infusion in 1 month as one cycle. 5-FU 5-fluorouracil

three planned dosage levels of 5-FU $(250,350$, and $450 \mathrm{mg} / \mathrm{m}^{2} /$ day) for 20 days (day $1-5,8-12,15-19$, and 22-26) by intravenous infusion from an implantable infusion port with an infusion device in 1 month (as one cycle) (Fig. 1). If none of the first three patients had dose-limiting toxicity (DLT), the dose was increased to the next dose level. If one or two of the first three patients had DLT, three additional patients were assigned to the same dose level; if only one or two of the six patients had DLT, the dose was increased to the next dose level; if all of the three patients or three or more of the six patients had DLT, the dose was defined as the MTD; the RD was defined as the level one step below the MTD. DLT was defined as the occurrence of any of the following toxicities: (1) hematologic toxicity of grade 4 ; (2) febrile $\left(\geq 38.5^{\circ} \mathrm{C}\right)$ neutropenia of grade 3; (3) thrombocytopenia of grade 3 that needed blood infusion for recovery; or (4) non-hematologic event of grade 3 .

\subsection{Safety Monitoring and Efficacy of Treatment}

Safety measurements included monitoring for adverse events (AEs) using the National Cancer Institute Common Toxicity Criteria, version 4.0 (NCI CTCAE v4.0) in one cycle. AEs from the use of combination therapy were monitored until recovery or remission. If toxicities associated with sorafenib occurred, the dose of sorafenib was reduced according to the drug information. Tumor response measurements, based on the Modified Response Evaluation Criteria in Solid Tumors (mRECIST) [38], were performed at baseline (within 21 days before initiation of treatment) and after one cycle (within 14 days of cessation or completion of treatment). The number and percentage of patients achieving complete response (CR), partial response (PR), stable disease (SD), and progressive disease (PD), and the disease control rate (DCR,\%) $(\mathrm{CR}+\mathrm{PR}+\mathrm{SD} / \mathrm{CR}+\mathrm{PR}+\mathrm{SD}+\mathrm{PD})$ were analyzed. 
For patients with SD or an objective response, follow-up assessments for survival were performed every 4 weeks until PD and/or death. After one cycle of combination therapy, sorafenib monotherapy was administered in consultation with the doctor in charge of the patient.

\subsection{Patients' Characteristics}

A total of 12 patients (all men; median age 65 years, age range 49-75 years) were enrolled in the study from September 2010 to September 2011. The performance status of all patients was 0 . The etiologies of HCC were $\mathrm{HBV}$ in four $(33.3 \%), \mathrm{HCV}$ in two $(16.7 \%)$, and others in six $(50 \%)$. The median maximum tumor size was $6.8 \mathrm{~cm}$ (range $1.0-9.6 \mathrm{~cm}$ ), and 11 patients $(91.7 \%)$ were Union for International Cancer Control (UICC) TNM stage IV-B. The number of patients was three $(25 \%)$ in cohort 1 , three $(25 \%)$ in cohort 2 , and six $(50 \%)$ in cohort 3 according to the $3+3$ design. The patients' characteristics are summarized in Table 1.

\section{Results}

\subsection{Evaluation of DLT and MTD}

The schedule of administration is shown in Fig. 1. There were three to six patients in each cohort. The first three patients started at a dose of $250 \mathrm{mg} / \mathrm{m}^{2}$ of 5 -FU and sorafenib $800 \mathrm{mg}$ daily (cohort 1); there were no DLTs observed during follow-up. The next three patients were treated with a dose of $350 \mathrm{mg} / \mathrm{m}^{2}$ of $5-\mathrm{FU}$ and sorafenib $800 \mathrm{mg}$ daily (cohort 2); there were no DLTs observed during follow-up. The next three patients were treated with a dose of $450 \mathrm{mg} / \mathrm{m}^{2}$ of 5-FU and sorafenib $800 \mathrm{mg}$ daily (cohort 3); there were two DLTs observed, and an

Table 1 Patients' baseline characteristics

\begin{tabular}{|c|c|c|c|c|}
\hline & Cohort 1 & Cohort 2 & Cohort 3 & Total (range or \%) \\
\hline 5-FU dose $\left(\mathrm{mg} / \mathrm{m}^{2}\right)$ & 250 & 350 & 450 & \\
\hline Number of patients & 3 & 3 & 6 & 12 \\
\hline Age or median age (range) (years) & $64,74,65$ & $74,49,55$ & $65(54-75)$ & $65(49-75)$ \\
\hline Sex: male, female & 3,0 & 3,0 & 6,0 & $12,0(100,0 \%)$ \\
\hline ECOG status: 0,1 & 3,0 & 3,0 & 6,0 & $12,0(100,0 \%)$ \\
\hline Etiology: HBV, HCV, others & $1,0,2$ & $2,0,1$ & $1,2,3$ & $4,2,6(33.3,16.7,50 \%)$ \\
\hline \multicolumn{5}{|l|}{ Radiological features (CT) } \\
\hline Median tumor diameter $(\mathrm{cm})$ & $5(1.3-7.3)$ & $7.9(2.5-9.6)$ & $6.8(1.0-9.1)$ & $6.8(1.0-9.6)$ \\
\hline Portal invasion & 0 & 0 & 1 (segmental) & $1(8.3 \%)$ \\
\hline \multicolumn{5}{|l|}{ UICC stage } \\
\hline I-II & 0 & 0 & 0 & 0 \\
\hline III & 0 & 0 & 1 & $1(8.3 \%)$ \\
\hline IV-A & 0 & 0 & 0 & 0 \\
\hline IV-B & 3 & 3 & 5 & $11(91.7 \%)$ \\
\hline \multicolumn{5}{|l|}{ Location of metastases } \\
\hline Lung & 2 & 2 & 1 & $5(41.7 \%)$ \\
\hline Lymph node & 1 & 2 & 3 & $6(50 \%)$ \\
\hline Bone & 1 & 1 & 1 & $3(25 \%)$ \\
\hline Others & 1 & 1 & 1 & $3(25 \%)$ \\
\hline Median AFP (ng/mL) & $4.2(3.0-5931.2)$ & $191.8(4.0-571.0)$ & $20.8(5.2-126.0)$ & $20.8(3.0-5931.2)$ \\
\hline Median PIVKA-II (mAU/mL) & $2130(763-23,199)$ & $11500(19-42,522)$ & $2480(23-110,648)$ & $2130(19-110,648)$ \\
\hline \multicolumn{5}{|l|}{ Prior treatments } \\
\hline None & 1 & 1 & 3 & $5(41.7 \%)$ \\
\hline Surgery & 1 & 0 & 2 & $3(25 \%)$ \\
\hline Ablation therapy & 0 & 0 & 0 & 0 \\
\hline TACE & 1 & 2 & 0 & $3(25 \%)$ \\
\hline HAIC & 0 & 0 & 1 & $1(8.3 \%)$ \\
\hline
\end{tabular}

5-FU 5-fluorouracil, AFP alpha-fetoprotein, $C T$ computed tomography, ECOG Eastern Cooperative Oncology Group, HAIC hepatic arterial infusion chemotherapy, $H B V$ hepatitis B virus, $H C V$ hepatitis $\mathrm{C}$ virus, PIVKA-II protein induced by vitamin $\mathrm{K}$ absence or antagonist II, TACE transcatheter arterial chemoembolization, TNM tumor, node, metastasis, UICC Union for International Cancer Control 
additional three patients were treated with the same dose. Ultimately, two of six patients in cohort 3 developed DLTs. Given these results, the MTD of 5-FU in combination therapy with sorafenib was $450 \mathrm{mg} / \mathrm{m}^{2}$, and $350 \mathrm{mg} / \mathrm{m}^{2}$ was defined as the RD for phase II study.

\subsection{Safety}

Table 2 shows the frequency of AEs. The non-hematologic AEs of any grade were fatigue $(50 \%)$, appetite loss $(50 \%)$, anorexia $(56 \%)$, vomiting $(8.3 \%)$, stomatitis $(33.3 \%)$, hand-foot skin reaction (HFSR) (58.3\%), skin rash $(8.3 \%)$, hypoxia $(8.3 \%)$, aspartate aminotransferase (AST) abnormality (25\%), alkaline phosphatase (ALP) abnormality $(8.3 \%)$, bilirubin abnormality $(16.7 \%)$, and hypoalbuminemia $(8.3 \%)$. The hematologic abnormalities of any grade were neutropenia (25\%) and thrombocytopenia (41.7\%). Febrile neutropenia and anemia were not observed. The observed grade 3 non-hematologic AEs were stomatitis (8.3\%) and HFSR (16.7\%). Only thrombocytopenia $(8.3 \%)$ was seen as a grade 3 hematologic AE. There were two DLTs observed in cohort 3 . One patient had grade 3 thrombocytopenia that needed blood infusion to recover, and the other patient had grade 3 stomatitis and grade 3 HFSR. Two patients needed dose reduction of sorafenib in cohort 2 . Their adherence rates to sorafenib were 75 and $87.5 \%$, respectively. Four patients needed dose reduction of sorafenib in cohort 3; three of them required the discontinuation of sorafenib for 1-2 week. Their adherence rates to sorafenib were $62.5 \%, 62.5,50$, and $50 \%$, respectively.

\subsection{Efficacy}

Ten patients were evaluated for tumor response after one cycle of treatment by mRECIST. Two DLT patients were evaluated at the time of discontinuation of treatment. Table 3 shows the summary of efficacy. Nine patients achieved SD (75\%), and three patients achieved PD (25\%). The DCR was $75 \%$. In detail, two patients in cohort 1 and one DLT patient in cohort 3 achieved PD.

Table 2 Adverse events

\begin{tabular}{|c|c|c|c|c|c|c|c|c|c|c|c|c|}
\hline & \multicolumn{4}{|c|}{ Cohort $1(n=3)$} & \multicolumn{4}{|c|}{ Cohort $2(n=3)$} & \multicolumn{4}{|c|}{ Cohort $3(n=6)$} \\
\hline & G1 & G2 & G3 & G4 & G1 & $\mathrm{G} 2$ & G3 & G4 & G1 & G2 & G3 & G4 \\
\hline \multicolumn{13}{|l|}{ Hematologic } \\
\hline Febrile neutropenia & - & - & - & - & - & - & - & - & - & - & - & - \\
\hline Neutropenia & 1 & - & - & - & - & 1 & - & - & - & 1 & - & - \\
\hline Anemia & - & - & - & - & - & - & - & - & - & - & - & - \\
\hline Thrombocytopenia & - & 1 & - & - & 1 & - & 1 & - & 2 & - & - & - \\
\hline \multicolumn{13}{|l|}{ Non-hematologic } \\
\hline Fatigue & 3 & - & - & - & - & - & - & - & - & - & - & - \\
\hline Nausea & - & - & - & - & - & - & - & - & - & - & - & - \\
\hline Appetite loss & - & 1 & - & - & - & - & - & - & 1 & - & - & - \\
\hline Vomiting & - & - & - & - & - & - & - & - & - & - & - & - \\
\hline Diarrhea & - & - & - & - & - & - & - & - & - & - & - & - \\
\hline Stomatitis & - & - & - & - & 1 & - & - & - & - & 2 & 1 & - \\
\hline Hand-foot skin reaction & - & 1 & - & - & - & 2 & - & - & - & 2 & 2 & - \\
\hline Skin rash & - & - & - & - & 1 & - & - & - & - & 1 & - & - \\
\hline Hypertension & - & - & - & - & - & - & - & - & - & - & - & - \\
\hline Hypoxia & - & 2 & - & - & - & - & - & - & - & - & - & - \\
\hline Hoarseness & - & - & - & - & - & - & - & - & 1 & - & - & - \\
\hline AST abnormality & 1 & - & - & - & 2 & - & - & - & -1 & - & - & - \\
\hline ALT abnormality & - & - & - & - & - & - & - & - & - & - & - & - \\
\hline ALP abnormality & 1 & - & - & - & - & - & - & - & - & - & - & - \\
\hline Bilirubin abnormality & - & - & - & - & 1 & - & - & - & 1 & - & - & - \\
\hline Hypoalbuminemia & - & - & - & - & 1 & - & - & - & - & - & - & - \\
\hline Epistaxis & - & - & - & - & - & - & - & - & - & 1 & - & - \\
\hline
\end{tabular}

Data are numbers

$A L T$ alanine aminotransferase, $A L P$ alkaline phosphatase, $A S T$ aspartate aminotransferase, $G$ grade 
Table 3 Efficacy

\begin{tabular}{llllll}
\hline & Number of patients & \multicolumn{4}{l}{ Response $n(\%)$} \\
\cline { 3 - 6 } & & CR & PR & SD & PD \\
\hline Cohort 1 & 3 & 0 & 0 & $1(33.3)$ & $2(66.7)$ \\
Cohort 2 & 3 & 0 & 0 & $3(100)$ & 0 \\
Cohort 3 & 6 & 0 & 0 & $5(58.3)$ & $1(16.7)$ \\
\hline
\end{tabular}

$C R$ complete response, $P D$ progressive disease, $P R$ partial response, $S D$ stable disease

\subsection{Case}

A 74-year-old man was diagnosed with moderately differentiated HCC at the time of hepatic resection 1 year before being enrolled in this study. Multiple metastases of HCC were observed on chest CT affecting both lungs before treatment (Fig. 2a). After combination therapy with sorafenib and 5-FU, the multiple lung metastases of HCC progressed, and a new lesion was detected at the right lobe of the lung (Fig. 2b). This case was evaluated as PD by mRECIST in this study. Six months after the beginning of treatment, multiple lung metastases of HCC showed PR, and the newly detected lesion disappeared (Fig. 2c).

\section{Discussion}

The aim of this study was to evaluate the safety and tolerability of combination therapy with sorafenib and cytotoxic agents. Furthermore, the aim was also to determine the RD of 5-FU for a phase II study. It has been reported that agents that inhibit VEGFR might enhance cytotoxic agents by normalizing tumor vasculature and decreasing the elevated interstitial pressure in tumors [35]. These effects were considered of value for HCC. In this study, intravenous infusion of 5-FU was combined with sorafenib. 5-FU monotherapy has been shown to be ineffective for advanced HCC. However, several drugs that are modulators that might increase 5-FU activity, when used in combination therapy with 5-FU, improved response rates or OS in advanced HCC patients in small phase II trials [16-18]. The methods of 5-FU administration were tested by various treatment schedules, dosages, and durations in the treatment of HCC $[8,14,15]$, and intravenous infusion of 5-FU used in the present study was found to be safe and tolerable. For that reason, we began to investigate the efficacy of combination therapy with sorafenib and cytotoxic agents using intravenous infusion of 5-FU. Several combination therapies with sorafenib and cytotoxic agents for advanced HCC have been reported. The combination
Fig. 2 A 74-year-old man diagnosed with moderately differentiated HCC. a Multiple lung metastases of HCC are observed before combination treatment on chest CT. b After one cycle, multiple metastases of HCC are enlarged and diagnosed as progressive disease on chest CT. c Six months after treatment, multiple metastases of HCC show partial response on chest CT. $C T$ computed tomography, $\mathrm{HCC}$ hepatocellular carcinoma
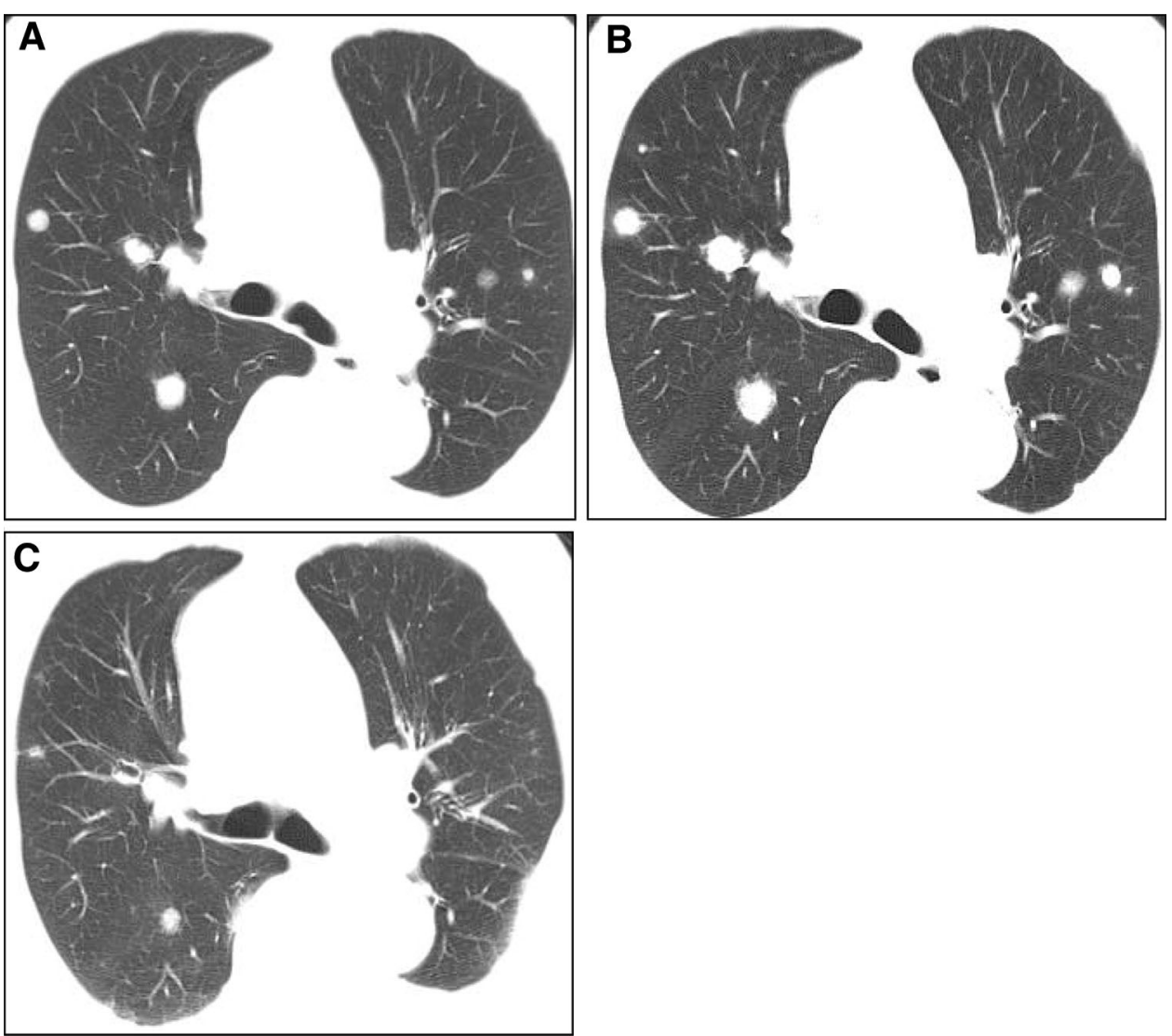
therapy with sorafenib and doxorubicin was found to have efficacy and tolerability for advanced HCC patients in a double-blind, phase II, multinational study [39]. Unfortunately, the treatment with sorafenib and doxorubicin did not improve OS compared with sorafenib alone in a phase III study [40]. Petrini et al. reported a phase II study of combination therapy with sorafenib and intravenous infusion of 5-FU [36]. Their treatment schedule was sorafenib $800 \mathrm{mg} /$ day and continuous infusion of $5-\mathrm{FU}(200 \mathrm{mg} /$ $\mathrm{m}^{2} /$ day) for days 1-14 (21-day cycles). The median TTP was 8 (95\% CI 5.7-10.4) months, and median OS was 13.7 (95\% CI 9.5-17.9) months. They concluded that combination therapy with sorafenib and intravenous infusion of 5-FU was safe and feasible. Moreover, high-dose intravenous infusion of 5-FU was reported to have manageable side effects in previous reports [16-18]. These facts encouraged us to perform combination therapy with sorafenib and high-dose 5-FU infusion for advanced HCC patients. Intravenous infusion of 5-FU $(250,350$, or $450 \mathrm{mg} / \mathrm{m}^{2} /$ day) for 20 days (day $1-5,8-12,15-19$, and 22-26) in 1 month as one cycle was selected.

In this study, four cases $(33.3 \%)$ of stomatitis occurred as AEs, and one of them had DLT (8.3\%). Stomatitis might occur frequently as a side effect of 5-FU, but this is often not the case as a side effect of sorafenib monotherapy. This is a critical AE for combination therapy and may need careful management in preparation for a phase II study.

The limitation of this study was that all patients were treated by intravenous 5-FU infusion for a long time and required 2 weeks hospitalization for monitoring DLT. In this study, we determined the RD of 5-FU in combination use with sorafenib, and we plan to treat study participants as outpatients in the next phase II study. The other limitation was the evaluation timing for response rate. As shown in the case, evaluation after one cycle might not be adequate for tumor response. With sorafenib monotherapy, a long duration of drug therapy improved TTP or OS, and longer periods of evaluation of tumor response might be needed with this combination therapy.

In conclusion, combination therapy with sorafenib and intravenous infusion of 5-FU for advanced HCC appears to be well-tolerated and may have the potential of therapeutic effects compared with sorafenib monotherapy.

\begin{abstract}
Acknowledgements The authors gratefully acknowledge the administrative support provided by Hatsumi Ueda, Terumi Hatakeyama, Megumi Kimura, and Tomoe Shimazaki and the technical support provided by Ayumi Kachi, Maki Makino, Masumi Kodama, Kazue Yoshida, and Keiko Sano.
\end{abstract}

\section{Compliance with Ethical Standards}

Conflict of interest Professor Naoya Sakamoto received lecture fees from Bristol Myers Squibb and Pharmaceutical K.K, and endowments from MSD K. K and Chugai Pharmaceutical Co., Ltd, and a research grant from Gilead Sciences, Inc. Dr Kenichi Morikawa received a research grant from Gilead Sciences. Inc. Dr Goki Suda received a research grant from Bristol Myers Squibb. Takuya Sho, Mitsuru Nakanishi, Masatsugu Ohara, Naoki Kawagishi, Jun Ito, Machiko Umemura, Takaaki Izumi, Masato Nakai, Koji Ogawa, Makoto Chuma, Takashi Meguro, Michio Nakamura, Atsushi Nagasaka, Hiromasa Horimoto, and Yoshiya Yamamoto declare that they have no conflict of interest.

Financial support This work was supported by Bayer Holding Ltd Japan as an endowment.

Trial registration number This study was registered at the UMIN Clinical Trials Registry as UMIN000004313.

Open Access This article is distributed under the terms of the Creative Commons Attribution-NonCommercial 4.0 International License (http://creativecommons.org/licenses/by-nc/4.0/), which permits any noncommercial use, distribution, and reproduction in any medium, provided you give appropriate credit to the original author(s) and the source, provide a link to the Creative Commons license, and indicate if changes were made.

\section{References}

1. El-Serag HB. Epidemiology of viral hepatitis and hepatocellular carcinoma. Gastroenterology. 2012;142(6):1264-73.

2. Torre LA, Bray F, Siegel RL, Ferlay J, Lortet-Tieulent J, Jemal A. Global cancer statistics, 2012. CA Cancer J Clin. 2015;65(2):87-108.

3. Torre LA, Siegel RL, Ward EM, Jemal A. Global cancer incidence and mortality rates and trends-an update. Cancer Epidemiol Biomark Prev. 2016;25(1):16-27.

4. Jun L, Zhenlin Y, Renyan G, Yizhou W, Xuying W, Feng X, et al. Independent factors and predictive score for extrahepatic metastasis of hepatocellular carcinoma following curative hepatectomy. Oncologist. 2012;17(7):963-9.

5. Lee HS. Management of patients with hepatocellular carcinoma and extrahepatic metastasis. Dig Dis. 2011;29(3):333-8.

6. Lee SW, Lee HL, Han NI, Jang JW, Bae SH, Choi JY, et al. Early treatment response to transcatheter arterial chemoembolization is associated with time to the development of extrahepatic metastasis and overall survival in intermediate-stage hepatocellular carcinoma. Cancer Chemother Pharmacol. 2017;79(1):81-8.

7. Kudo M, Matsui O, Izumi N, Iijima H, Kadoya M, Imai Y, et al. JSH consensus-based clinical practice guidelines for the management of hepatocellular carcinoma: 2014 update by the Liver Cancer Study Group of Japan. Liver Cancer. 2014;3(3-4): 458-68.

8. Gong XL, Qin SK. Progress in systemic therapy of advanced hepatocellular carcinoma. World J Gastroenterol. 2016;22(29): 6582-94.

9. Petrelli F, Coinu A, Borgonovo K, Cabiddu M, Ghilardi M, Lonati V, et al. Oxaliplatin-based chemotherapy: a new option in advanced hepatocellular carcinoma. A systematic review and pooled analysis. Clin Oncol. 2014;26(8):488-96.

10. Patrikidou A, Sinapi I, Regnault H, Fayard F, Bouattour M, Fartoux L, et al. Gemcitabine and oxaliplatin chemotherapy for advanced hepatocellular carcinoma after failure of anti-angiogenic therapies. Investig New Drugs. 2014;32(5):1028-35.

11. Qin S, Bai Y, Lim HY, Thongprasert S, Chao Y, Fan J, et al. Randomized, multicenter, open-label study of oxaliplatin plus fluorouracil/leucovorin versus doxorubicin as palliative 
chemotherapy in patients with advanced hepatocellular carcinoma from Asia. J Clin Oncol. 2013;31(28):3501-8.

12. Qin S, Cheng Y, Liang J, Shen L, Bai Y, Li J, et al. Efficacy and safety of the FOLFOX4 regimen versus doxorubicin in Chinese patients with advanced hepatocellular carcinoma: a subgroup analysis of the EACH study. Oncologist. 2014;19(11):1169-78.

13. Zaanan A, Williet N, Hebbar M, Dabakuyo TS, Fartoux L, Mansourbakht T, et al. Gemcitabine plus oxaliplatin in advanced hepatocellular carcinoma: a large multicenter AGEO study. J Hepatol. 2013;58(1):81-8.

14. Ishikawa T. Chemotherapy with enteric-coated tegafur/uracil for advanced hepatocellular carcinoma. World J Gastroenterol. 2008;14(18):2797-801.

15. Ishikawa T, Ichida T, Sugitani S, Tsuboi Y, Genda T, Sugahara S, et al. Improved survival with oral administration of enteric-coated tegafur/uracil for advanced stage IV-A hepatocellular carcinoma. J Gastroenterol Hepatol. 2001;16(4):452-9.

16. Damdinsuren B, Nagano H, Monden M. Combined intra-arterial 5-fluorouracil and subcutaneous interferon-alpha therapy for highly advanced hepatocellular carcinoma. Hepatol Res. 2007;37(Suppl 2):S238-50.

17. Ikeda M, Okusaka T, Ueno H, Takezako Y, Morizane C. A phase II trial of continuous infusion of 5-fluorouracil, mitoxantrone, and cisplatin for metastatic hepatocellular carcinoma. Cancer. 2005;103(4):756-62.

18. Tanioka H, Tsuji A, Morita S, Horimi T, Takamatsu M, Shirasaka $\mathrm{T}$, et al. Combination chemotherapy with continuous 5-fluorouracil and low-dose cisplatin infusion for advanced hepatocellular carcinoma. Anticancer Res. 2003;23(2C):1891-7.

19. Wilhelm SM, Carter C, Tang L, Wilkie D, McNabola A, Rong H, et al. BAY 43-9006 exhibits broad spectrum oral antitumor activity and targets the RAF/MEK/ERK pathway and receptor tyrosine kinases involved in tumor progression and angiogenesis. Cancer Res. 2004;64(19):7099-109.

20. Llovet JM, Ricci S, Mazzaferro V, Hilgard P, Gane E, Blanc JF, et al. Sorafenib in advanced hepatocellular carcinoma. N Engl J Med. 2008;359(4):378-90.

21. Cheng AL, Kang YK, Chen Z, Tsao CJ, Qin S, Kim JS, et al. Efficacy and safety of sorafenib in patients in the Asia-Pacific region with advanced hepatocellular carcinoma: a phase III randomised, double-blind, placebo-controlled trial. Lancet Oncol. 2009;10(1):25-34.

22. Verslype C, Van Cutsem E, Dicato M, Arber N, Berlin JD, Cunningham D, et al. The management of hepatocellular carcinoma. Current expert opinion and recommendations derived from the 10th World Congress on Gastrointestinal Cancer, Barcelona, 2008. Ann Oncol. 2009;20(Suppl 7):vii1-6.

23. Chuma M, Terashita K, Sakamoto N. New molecularly targeted therapies against advanced hepatocellular carcinoma: from molecular pathogenesis to clinical trials and future directions. Hepatol Res Off J Jpn Soc Hepatol. 2015;45(10):E1-11.

24. Ikemoto T, Shimada M, Yamada S. Pathophysiology of recurrent hepatocellular carcinoma after radiofrequency ablation. Hepatol Res. 2017;47(1):23-30.

25. Llovet JM, Villanueva A, Lachenmayer A, Finn RS. Advances in targeted therapies for hepatocellular carcinoma in the genomic era. Nat Rev Clin Oncol. 2015;12(7):408-24.

26. Johnson PJ, Qin S, Park JW, Poon RT, Raoul JL, Philip PA, et al. Brivanib versus sorafenib as first-line therapy in patients with unresectable, advanced hepatocellular carcinoma: results from the randomized phase III BRISK-FL study. J Clin Oncol. 2013;31(28):3517-24.

27. Llovet JM, Decaens T, Raoul JL, Boucher E, Kudo M, Chang C, et al. Brivanib in patients with advanced hepatocellular carcinoma who were intolerant to sorafenib or for whom sorafenib failed: results from the randomized phase III BRISK-PS study. J Clin Oncol. 2013;31(28):3509-16.

28. Cheng AL, Kang YK, Lin DY, Park JW, Kudo M, Qin S, et al. Sunitinib versus sorafenib in advanced hepatocellular cancer: results of a randomized phase III trial. J Clin Oncol. 2013;31(32):4067-75.

29. Cainap C, Qin S, Huang WT, Chung IJ, Pan H, Cheng Y, et al. Linifanib versus sorafenib in patients with advanced hepatocellular carcinoma: results of a randomized phase III trial. J Clin Oncol. 2015;33(2):172-9.

30. Zhu AX, Park JO, Ryoo BY, Yen CJ, Poon R, Pastorelli D, et al. Ramucirumab versus placebo as second-line treatment in patients with advanced hepatocellular carcinoma following first-line therapy with sorafenib (REACH): a randomised, double-blind, multicentre, phase 3 trial. Lancet Oncol. 2015;16(7):859-70.

31. Zhu AX, Kudo M, Assenat E, Cattan S, Kang YK, Lim HY, et al. Effect of everolimus on survival in advanced hepatocellular carcinoma after failure of sorafenib: the EVOLVE-1 randomized clinical trial. Jama. 2014;312(1):57-67.

32. Zhu AX, Rosmorduc O, Evans TR, Ross PJ, Santoro A, Carrilho FJ, et al. SEARCH: a phase III, randomized, double-blind, placebo-controlled trial of sorafenib plus erlotinib in patients with advanced hepatocellular carcinoma. J Clin Oncol. 2015;33(6):559-66.

33. Chao Y, Li CP, Chau GY, Chen CP, King KL, Lui WY, et al. Prognostic significance of vascular endothelial growth factor, basic fibroblast growth factor, and angiogenin in patients with resectable hepatocellular carcinoma after surgery. Ann Surg Oncol. 2003;10(4):355-62.

34. Poon RT, Ho JW, Tong CS, Lau C, Ng IO, Fan ST. Prognostic significance of serum vascular endothelial growth factor and endostatin in patients with hepatocellular carcinoma. Br J Surg. 2004;91(10):1354-60.

35. Jain RK. Normalizing tumor vasculature with anti-angiogenic therapy: a new paradigm for combination therapy. Nat Med. 2001;7(9):987-9.

36. Petrini I, Lencioni M, Ricasoli M, Iannopollo M, Orlandini C, Oliveri $\mathrm{F}$, et al. Phase II trial of sorafenib in combination with 5-fluorouracil infusion in advanced hepatocellular carcinoma. Cancer Chemother Pharmacol. 2012;69(3):773-80.

37. Bruix J, Sherman M, American Association for the Study of Liver Diseases. Management of hepatocellular carcinoma: an update. Hepatology. 2011;53(3):1020-2.

38. Lencioni R, Llovet JM. Modified RECIST (mRECIST) assessment for hepatocellular carcinoma. Semin Liver Dis. 2010;30(1):52-60.

39. Abou-Alfa GK, Johnson P, Knox JJ, Capanu M, Davidenko I, Lacava J, et al. Doxorubicin plus sorafenib vs doxorubicin alone in patients with advanced hepatocellular carcinoma: a randomized trial. Jama. 2010;304(19):2154-60.

40. Abou-Alfa GK, Niedzwieski D, Knox JJ, Kaubisch A, Posey J, Tan BR et al. Phase III randomized study of sorafenib plus doxorubicin versus sorafenib in patients with advanced hepatocellular carcinoma (HCC): CALGB 80802 (Alliance). J Clin Oncol. 2016;34(suppl 4S; abstr 192). 